Arq. Bras. Med. Vet. Zootec., v.73, n.2, p.451-459, 2021

\title{
Fatores de influência sobre o desempenho reprodutivo em vacas leiteiras
}

[Influence factors on reproductive performance in dairy cows]

\author{
S.R.V. Soares ${ }^{1}$, R.B. Reis $^{2}$, A.N. Dias $^{1 *}$
}

${ }^{1}$ Faculdade Rehagro - Belo Horizonte, MG

${ }^{2}$ Universidade Federal de Minas Gerais - Belo Horizonte, MG

\section{RESUMO}

O objetivo deste estudo foi avaliar os efeitos das estações do parto e da inseminação, da retenção de placenta, da natimortalidade e da ordem de parto sobre o desempenho reprodutivo de vacas leiteiras. Utilizou-se a regressão logística, em que as variáveis dependentes foram concepção à primeira inseminação pós-parto e percentual de vacas prenhes no rebanho aos 100, 150 ou 200 DEL e as variáveis independentes foram a estação do parto e a estação da inseminação, a retenção de placenta, a natimortalidade e a ordem de parto. A estação do parto e a estação da inseminação influenciaram o desempenho reprodutivo, reduzindo a fertilidade das vacas nas estações quentes do ano. $\mathrm{O}$ atraso na primeira inseminação aumentou a concepção na primeira inseminação, mas reduziu o percentual de vacas prenhes ao longo da lactação. O desempenho reprodutivo da vaca leiteira foi afetado por vários fatores, relacionados à vaca, ao ambiente e ao manejo a ela imposto.

Palavras-chave: reprodução, ambiente, bovinocultura leiteira, prenhes

\begin{abstract}
The objective of this study was to evaluate the effects of the calving season, insemination, placenta retention, stillbirth and birth order on the reproductive performance of dairy cows. Logistic regression was used, where the dependent variables were conception at the first postpartum insemination and the percentage of cows pregnant in the herd at 100, 150 and 200 DEL. The independent variables were delivery season and insemination season, placenta retention, stillbirth and delivery order. The calving season and the insemination season influenced reproductive performance, and in the hot seasons of the year the fertility of the cows was reduced. The delay in the first insemination increased conception in the first insemination but reduced the percentage of pregnant cows during lactation. The reproductive performance of the dairy cow was affected by several factors, related to the cow, the environment and the management imposed on it.
\end{abstract}

Keywords: reproduction, environment, dairy cattle production, pregnant

\section{INTRODUÇÃO}

A realidade da indústria láctea é a redução no número de fazendas e o aumento da produção. $\mathrm{O}$ rápido progresso genético e das práticas de manejo elevou a produção de leite das vacas no mundo. No entanto, a consolidação dessa nova estrutura da indústria leiteira criou vários novos desafios; talvez o maior deles seja a redução na eficiência reprodutiva da vaca leiteira moderna. Alta eficiência reprodutiva é um pré-requisito para garantir a lucratividade na pecuária de leite, pois a vaca precisa parir para dar início à nova lactação.

Estudos afirmam que existe antagonismo entre produção de leite e desempenho reprodutivo, e o aumento na produção de leite tem sido responsabilizado pela queda no desempenho reprodutivo (Bello et al., 2012). No entanto, fatores como produção de leite, escore de condição corporal, saúde no pós-parto, raça, ordem de parto, dias em lactação, regime alimentar e sazonalidade têm efeitos

Recebido em 11 de setembro de 2019

Aceito em 9 de março de 2020

*Autor para correspondência (corresponding author)

E-mail: adelio.dias@ rehagro.com.br 
consideráveis na eficiência reprodutiva da vaca leiteira (Berry et al., 2003; Tsuruta, et. al., 2009). O problema da redução na eficiência reprodutiva é provavelmente uma combinação de vários fatores fisiológicos e de manejo.

Este estudo teve como objetivo avaliar se o desempenho reprodutivo de vacas leiteiras que ficaram gestantes ou não até 100, 150 ou 200 DEL foi afetado pela estação do parto, pela estação da inseminação, pela retenção de placenta, pela natimortalidade e pela ordem do parto.

\section{MATERIAL E MÉTODOS}

O banco de dados utilizado foi disponibilizado pela empresa Ideagri $^{\odot}$. Foram selecionados rebanhos com, no mínimo, 100 lactações por ano, nos anos de 2009 a 2011. Desse banco de dados inicial, foram retirados dados inconsistentes. As inconsistências tratadas foram: lactações com produção acumulada aos 60 dias igual a zero, lactações com dois controles leiteiros ou menos, lactações com intervalo entre controles leiteiros maior que 45 dias, lactações iniciadas por aborto, lactações com o período de controle leiteiro menor que $80 \%$ em relação à duração da lactação, fazendas sem anotação de retenção de placenta e natimorto. Os dados contêm serviços realizados por inseminação artificial, monta natural e com uso de protocolos de sincronização de ovulação.

O banco de dados utilizado para o estudo da situação reprodutiva aos 100, 150 e 200 dias de lactação foi constituído de 16.948 lactações e 17 rebanhos, em sistema de confinamento ou sistema de pastejo nas águas e confinamento na seca, localizados em Minas Gerais (13), Paraná (2), São Paulo (1) e Goiás (1). O banco de dados para o estudo da concepção à primeira inseminação foi formado a partir do banco de dados descritos acima ( $n=15874$ lactações), sendo retiradas apenas as inseminações sem resultado de diagnóstico de gestação.

A análise estatística foi realizada utilizando-se o sistema de análises estatísticas SAS (Statistical..., 2006). As variáveis binomiais foram analisadas por regressão logística com eliminação stepwise, pelo procedimento LOGISTIC do SAS. A equação de regressão logística utilizada foi:

$$
\log (\mathrm{p} / 1-\mathrm{p})=\mathrm{a}+\beta_{\mathrm{n}} \mathrm{X}_{\mathrm{n}}
$$

em que: $\mathrm{p}=$ probabilidade de ocorrência do evento estudado; $(\mathrm{p}-1)=$ probabilidade de não ocorrência do evento estudado; $\alpha=$ coeficiente de regressão estimado para o intercepto; $\beta \mathrm{n}=$ coeficiente de regressão da variável independente $\mathrm{n} ; \mathrm{x}=$ valor $\mathrm{da}$ variável independente $\mathrm{n}$.

As variáveis dependentes estudadas foram concepção (sim ou não) na primeira inseminação pós-parto e situação reprodutiva (prenhe ou vazia) aos 100, 150 e 200 DEL. As variáveis independentes consideradas na análise estatística foram o ano de parto, a estação do parto, a natimortalidade, a retenção de placenta, a ordem de parto e a estação da inseminação. A variável estação da inseminação foi considerada apenas na regressão logística para a variável dependente concepção à primeira inseminação. A variável independente tipo de cobertura não foi incluída no modelo, pois $100 \%$ dos primeiros serviços foram realizados utilizando-se inseminação artificial.

As estações do parto e da inseminação foram classificadas em verão (dezembro, janeiro e fevereiro), outono (março, abril e maio), primavera (setembro, outubro e novembro) e inverno (junho, julho e agosto). Natimorto foi classificado como cria nascida morta ou que morreu até 24 horas após o parto. A retenção de placenta foi classificada como a não expulsão dos anexos fetais até o dia seguinte ao parto.

Fazendas que forneciam dieta total no cocho durante o ano todo foram consideradas sistema confinado. Já as que forneciam dieta total no cocho durante a estação seca e pasto com suplementação concentrada no cocho durante estação chuvosa foi denominada pasto. A variável independente fazenda foi mantida para ajuste do modelo, mas não foi objeto deste estudo.

A regressão logística foi utilizada quando a variável resposta era dicotômica, ou seja, tem 
valor zero ou um. No modelo de regressão logística, utiliza-se uma série de variáveis independentes para predizer a ocorrência de um determinado evento. A probabilidade de ocorrência do evento foi obtida pela fórmula:

$\mathrm{P}(\mathrm{y})=11+e-(\alpha+\Sigma \beta 1 X 1) \quad($ Hosmer $\mathrm{e}$ Lemeshow, 1985).

Os termos $\alpha$ e $\beta 1$ no modelo são parâmetros desconhecidos e são estimados pelo método de máxima verossimilhança a partir dos dados amostrais obtidos. $\mathrm{Na}$ regressão logística, os resultados são discutidos em termos de odds ratio. O odds ratio da ocorrência de um evento é a relação esperada entre a probabilidade de ocorrência de um evento pela probabilidade de não ocorrência de um evento. Um valor de odds ratio de quatro significa que a probabilidade de o evento ocorrer é quatro vezes maior do que a de o evento não ocorrer. A equação para o cálculo do odds ratio é demonstrada abaixo.

Odds ratio $=$ probabilidade de ocorrência do evento probabilidade de não ocorrência do evento.

Dessa forma, são formados ni $x$ nj pares de observações (sucesso x insucesso), classificados em concordantes quando $\mathrm{Pi}>\mathrm{Pj}$, discordantes quando $\mathrm{Pi}<\mathrm{Pj}$ e empatados se $\mathrm{Pi}=\mathrm{Pj}$ (Bergmann e Hohenboken, 1992).

\section{RESULTADOS E DISCUSSÃO}

A frequência de partos ocorridos foi maior no outono e no inverno (Tab. 1). Isso é explicado pelo fato de que normalmente a taxa de concepção aumenta nas estações frias do ano (Huang et al., 2009), portanto os partos tendem a se concentrar no outono e no inverno. Além disso, algumas das fazendas estudadas realizam parte das inseminações das novilhas entre os meses de maio e outubro e, consequentemente, os partos ocorrerão entre fevereiro e julho.

A incidência de natimorto observada, 5,0\% (Tab. 1), está dentro da faixa esperada para rebanhos leiteiros, resultado semelhante ao estudo realizado com um rebanho em sistema de pastejo em Minas Gerais, cuja incidência foi de 5,3\% (Nobre et al., 2012).
A incidência de retenção de placenta observada foi de 12,0\% (Tab.1). Esse valor ficou dentro da faixa de variação observada em outros estudos, de $3,8 \%$ a $30 \%$ (Laven e Peters, 1996). Nobre et al. (2012) observaram incidência de retenção de placenta de $12,8 \%$ em um rebanho em produção sob sistema de pastejo no estado de Minas Gerais, Brasil. A incidência de retenção de placenta no presente estudo ficou no limite inferior da faixa de variação, significando que o banco de dados estudado reflete a realidade de rebanhos com baixa incidência de retenção de placenta.

Tabela 1. Frequências observadas das lactações analisadas por regressão logística quanto à concepção à primeira inseminação segundo a estação do ano, ocorrências ao parto, ordem do parto e sistema de criação de vacas leiteiras paridas de 2009 a 2011

\begin{tabular}{lcc}
\multicolumn{1}{c}{ Variável } & $\mathrm{n}$ & $\%$ \\
\hline Estação do parto & & \\
Outono & 4.902 & 35,9 \\
Inverno & 3.901 & 28,6 \\
Primavera & 2.589 & 19,0 \\
Verão & 2.253 & 16,5 \\
& & \\
Natimorto & & \\
Não & 12.958 & 95,0 \\
Sim & 687 & 5,0 \\
& & \\
Retenção de placenta & & \\
Não & 12.001 & 88,0 \\
Sim & 1.644 & 12,0 \\
& & \\
Ordem de parto & & \\
1 & 4.825 & 35,4 \\
2 & 3.541 & 26,0 \\
$>=3$ & 5.279 & 38,7 \\
Sistema & & \\
Confinado & & \\
Pasto & 4.895 & 35,9 \\
\hline
\end{tabular}

A taxa de concepção na primeira inseminação foi de $35,8 \%$ (Tab. 2). Este resultado é inferior aos valores encontrados na literatura, que são acima de $40 \%$ para a taxa de concepção à primeira inseminação (Melendez e Pinedo, 2007; Inchaisri et al., 2010). No entanto, Teixeira (2010) encontrou taxa de concepção na primeira inseminação de $26 \%$ em estudo com um rebanho Holandês criado em sistema confinado em São Paulo, Brasil. 
Tabela 2. Taxas de concepção à primeira inseminação e porcentagens de vacas leiteiras gestantes aos 100, 150 e 200 dias em lactação, com produções de 2.506 a $16.821 \mathrm{~kg}$ de leite corrigidas para 305 dias, de vacas paridas de 2009 a 2011

\begin{tabular}{lc}
\hline \multicolumn{1}{c}{ Variável independente } & $\%$ \\
\hline Concepção na primeira inseminação & 35,3 \\
Vacas prenhes aos 100 DEL & 40,2 \\
Vacas prenhes aos 150 DEL & 58,3 \\
Vacas prenhes aos 200 DEL & 68,1 \\
\hline
\end{tabular}

Os resultados das análises de regressão logística multivariada para o coeficiente $\beta$, erro-padrão e valor de $\mathrm{P}$ de cada variável regressora estão demonstrados na Tab. 3. Os resultados de $\%$ de sucesso na primeira inseminação, \% de prenhes aos 100, 150 e 200 dias de lactação, odds ratio e intervalo de confiança a $95 \%$ do odds ratio para cada variável regressora estão demonstrados na Tab. 4.

Tabela 3. Coeficiente de regressão $\beta$, erro-padrão (ep) e P valor das análises de regressão logística multivariada dos fatores de risco para sucesso à primeira inseminação ( $\left.1^{\mathrm{a}} \mathrm{IA}\right)$ e situação reprodutiva aos 100 (SR100), 150 (SR150) e 200 (SR200) dias de lactação de vacas leiteiras paridas entre 2009 e 2011

\begin{tabular}{|c|c|c|c|c|c|c|c|c|c|c|c|c|}
\hline \multirow{2}{*}{ Parâmetro } & \multicolumn{3}{|c|}{$\mathrm{TC} 1^{\circ} \mathrm{IA}$} & \multicolumn{3}{|c|}{ SR 100} & \multicolumn{3}{|c|}{ SR 150} & \multicolumn{3}{|c|}{ SR 200} \\
\hline & $\beta$ & ep & $\mathrm{P}$ & $\beta$ & ep & $\mathrm{P}$ & $\beta$ & ep & $\mathrm{P}$ & $\beta$ & ep & $\mathrm{P}$ \\
\hline Outono & Ref. & & & Ref. & & & Ref. & & & Ref. & & \\
\hline Inverno & 0,000907 & 0,0404 & 0,9821 & $-0,0422$ & 0,0319 & 0,1857 & $-0,1202$ & 0,0310 & 0,0001 & $-0,1440$ & 0,0324 & $<0,0001$ \\
\hline Primavera & 0,0633 & 0,0492 & 0,1981 & $-0,0357$ & 0,0364 & 0,3266 & $-0,1832$ & 0,0352 & $<0,0001$ & $-0,2557$ & 0,0364 & $<0,0001$ \\
\hline Verão & $-0,1419$ & 0,0475 & 0,0028 & $-0,1554$ & 0,0403 & 0,0001 & 0,0224 & 0,0375 & 0,5506 & 0,1682 & 0,0397 & $<0,0001$ \\
\hline \multicolumn{13}{|c|}{ Estação da $1^{\mathrm{a}}$ inseminação } \\
\hline Outono & Ref. & & & & & & & & & & & \\
\hline Inverno & 0,1419 & 0,0378 & 0,0002 & & & & & & & & & \\
\hline Primavera & 0,0680 & 0,0420 & 0,1051 & & & & & & & & & \\
\hline Verão & $-0,0939$ & 0,0506 & 0,0636 & & & & & & & & & \\
\hline \multicolumn{13}{|c|}{ Natimorto } \\
\hline Não & Ref. & & & Ref. & & & Ref. & & & Ref. & & \\
\hline Sim & $-0,2506$ & 0,0608 & $<0,0001$ & $-0,3964$ & 0,0626 & $<0,0001$ & $-0,3823$ & 0,0566 & $<0,0001$ & $-0,3273$ & 0,0574 & $<0,0001$ \\
\hline \multicolumn{13}{|c|}{ Ordem do parto } \\
\hline 1 & Ref. & & & Ref. & & & Ref. & & & Ref. & & \\
\hline 2 & $-0,00210$ & 0,0289 & 0,9419 & 0,000046 & 0,0285 & 0,9987 & 0,00753 & 0,0280 & 0,7879 & 0,0208 & 0,0295 & 0,4797 \\
\hline$\geq 3$ & $-0,1528$ & 0,0277 & $<0,0001$ & $-0,1486$ & 0,0268 & $<0,0001$ & $-0,1771$ & 0,0258 & $<0,0001$ & $-0,1808$ & 0,0271 & $<0,0001$ \\
\hline
\end{tabular}

A probabilidade de concepção à primeira inseminação das vacas que pariram no verão foi menor $(\mathrm{OR}=0,803 ; \mathrm{P}<0,01)$ do que de vacas que pariram no outono. Vacas que pariram no inverno e na primavera não tiveram diferença na probabilidade de concepção à primeira inseminação das vacas que pariram no outono. Não houve diferença entre as inseminações realizadas no verão e na primavera em relação às inseminações realizadas no outono. No inverno, a probabilidade de sucesso à primeira inseminação foi maior $(\mathrm{OR}=1,130 ; \mathrm{P}<0,01)$ comparada à que houve no outono.

O efeito da estação do parto no percentual de vacas prenhes aos 100, 150 e 200 DEL foi variado. Efeito negativo do verão sobre a reprodução foi observado apenas no percentual de vacas prenhes aos 100 DEL. As vacas que pariram no verão tiveram a probabilidade de prenhez reduzida em $32,2 \%$ em relação às vacas que pariram no outono. Aos 150 e 200 DEL, não houve diferença na probabilidade de prenhez para as vacas que pariram no verão e no outono. Vacas que pariram no inverno e na primavera não tiveram probabilidade de prenhez diferente das que pariram no outono aos 100 DEL. Aos 150 e 200 DEL, as vacas que pariram no inverno tiveram probabilidade de prenhez reduzida em $43,3 \%$ e $33,8 \%$, respectivamente. Já as vacas que pariram na primavera tiveram probabilidade de prenhez reduzida em $46,3 \%$ e $37,4 \%$ aos 150 e 200 DEL, respectivamente. 
Tabela 4. Percentual de sucesso à $1^{\mathrm{a}}$ inseminação (\% Suc.), percentual de prenhes (\% prenhes), odds ratio (OR) e intervalo de confiança com 95\% de confiança (IC - 95\% Wald) das análises de regressão logística multivariada dos fatores de risco para concepção à primeira inseminação (TC $1^{\mathrm{a}}$ IA) e situação reprodutiva aos 100 (SR100), 150 (SR150) e 200 (SR200) dias de lactação de vacas leiteiras paridas entre os anos de 2009 e 2011

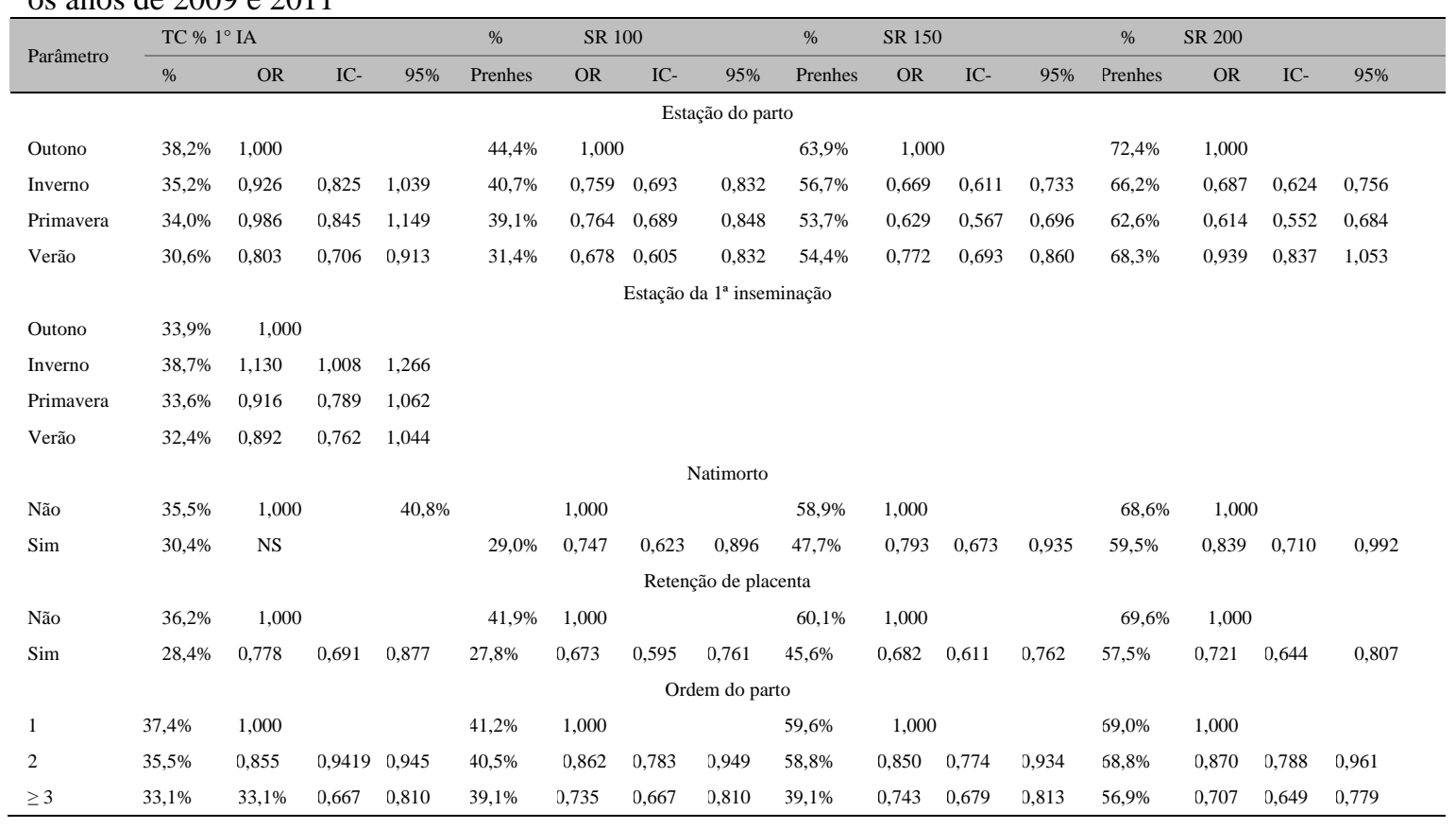

Fica claro que existe interação entre a estação do parto e os dias em lactação para a probabilidade de prenhez. Essa interação é observada na Fig. 1, onde as curvas de sobrevivência não se mantêm paralelas, mas se cruzam ao longo do gráfico. $\mathrm{O}$ efeito do verão é mais importante no início da lactação. Para vacas que parem no verão, o período de inseminação inicial ocorre no verão, mas posteriormente elas serão inseminadas no outono e no inverno, e isso pode justificar por que elas têm a mesma probabilidade de prenhez aos 200 DEL, comparadas às vacas que parem no outono. As vacas que parem na primavera e no inverno reduziram a probabilidade de prenhez ao longo da lactação em relação às vacas que pariram no outono. Isso pode estar relacionado à estação em que elas são inseminadas, pois, para as vacas que parem no inverno e na primavera, o período de inseminação ocorre, em grande parte, nas estações quentes do ano. A estação de parto outono obteve os melhores resultados tanto para a concepção à primeira inseminação quanto para a probabilidade de prenhez ao longo da lactação, pois as vacas conceberam em épocas de temperaturas amenas.

Outros trabalhos encontraram efeito significativo da estação do parto e estação da inseminação sobre o desempenho reprodutivo (De Vries e Risco, 2005; Melendez e Pinedo, 2007; Huang et al., 2009). Em regiões de clima tropical e subtropical, o estresse térmico é uma das principais causas da queda na fertilidade de vacas leiteiras (Huang et al., 2009). No entanto, Teixeira (2010) não observou efeito da estação da inseminação no desempenho reprodutivo em um rebanho Holandês em sistema confinado tipo free stall.

O efeito da estação do parto sobre o desempenho reprodutivo parece estar relacionado às condições climáticas, particularmente porque é observada redução no desempenho reprodutivo em períodos nos quais a temperatura ambiente é normalmente alta. 


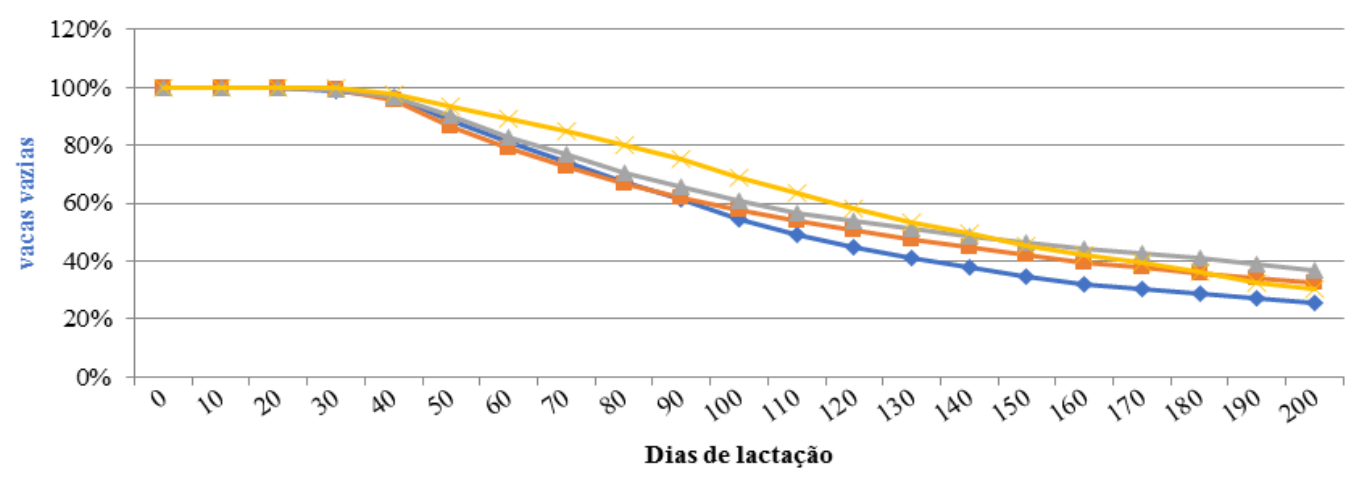

$\longrightarrow$ Outono —-Invemo —-Primavera $\longrightarrow$ Verão

Figura 1. Curva de sobrevivência para prenhez vs. estação do parto.

Vacas sob estresse térmico apresentaram redução na duração e na intensidade do estro (Dransfield et al., 1998), aumento da incidência de anestro e cio silencioso (Gwazdauskas et al., 1981). A redução da manifestação de estro diminui a probabilidade de a vaca ser inseminada e aumenta a probabilidade de ela ser inseminada no momento errado, uma vez que a duração do estro é menor. Isso porque ocorre redução da qualidade dos oócitos (Sartori et al., 2002; Torres-Júnior et al., 2008; Baumgard e Rhoads, 2013), redução no desenvolvimento da competência do oócito (Hansen, 2004) e redução na sobrevivência embrionária (Paula-Lopes et al., 2003; Hansen, 2004). Esses fatores explicam a queda na concepção à primeira inseminação e a probabilidade de prenhez aos 100 DEL.

Outro fator é o aumento da incidência de doenças durante o período de estresse térmico (DuBois e Willians, 1980). Como foi demonstrado neste estudo, o aumento da incidência de retenção de placenta e de natimorto reduz o desempenho reprodutivo. Portanto, o conjunto de alterações provocadas pelo estresse térmico pode ter reduzido a eficiência reprodutiva das vacas que pariram nas estações quentes do ano. Em país tropical, onde as temperaturas médias são elevadas, o fator estação do parto é importante no desempenho reprodutivo da vaca leiteira. Portanto, é fundamental investir no resfriamento dos animais, a fim de se melhorar o desempenho reprodutivo dos animais.

A concepção à primeira inseminação de vacas que tiveram natimorto foi de $30,1 \%$, e para as vacas que não tiveram natimorto foi de $36,1 \%$ (Fig. 3). A ocorrência de natimorto não teve efeito sobre a concepção à primeira inseminação. A concepção à primeira inseminação das vacas que tiveram retenção de placenta foi de $28,4 \%$, e para as vacas que não tiveram foi de $36,2 \%$. A ocorrência de retenção de placenta reduziu o risco de concepção na primeira inseminação em $22,2 \%$. As ocorrências de natimorto e de retenção de placenta também reduziram o risco de prenhez aos 100, 150 e 200 DEL. Vacas que não apresentaram retenção de placenta ou natimorto possuíram $32,7 \%$ e $25,3 \%$, respectivamente, mais chances de se tornarem prenhes até 100 DEL. Nas Fig. 2 e 3, observamse que os efeitos negativos da natimortalidade e da retenção de placenta duram até aproximadamente 200 dias após o parto.

A retenção de placenta reduz a concepção ao primeiro serviço, aumenta o tempo pós-parto para a concepção e aumenta o número de serviços por concepção (Gröhn e Rajala-Schultz, 2000; Nobre et al., 2012). E o efeito da retenção de placenta no desempenho reprodutivo está associado com o aumento de metrites (Gröhn e Rajala-Schultz, 2000) e de outras desordens no pós-parto, como mastite e cetose. A retenção de placenta é sintoma de período de transição subótimo, mas somente é importante para o desempenho reprodutivo por ser um fator de risco para a ocorrência de metrite e endometrite (LeBlanc, 2008).

Esses resultados são consistentes com a literatura consultada (Berry et al., 2007; Inchaisri et al., 2010; Nobre et al., 2012). A redução no desempenho reprodutivo causada pela retenção de placenta parece ocorrer de forma indireta (LeBlanc, 2008), pelo aumento na ocorrência de 
outras desordens (Gröhn e Rajala-Schultz, 2000; LeBlanc, 2008). Gröhn e Rajala-Schultz (2000) demonstraram que vacas com retenção de placenta têm $340 \%$ e $150 \%$ maiores probabilidades de desenvolverem metrite no pósparto recente e tardio, respectivamente. Esse aumento na ocorrência de metrite é a principal rota pela qual a retenção de placenta afeta a fertilidade (Laven e Peters, 1996).
O acompanhamento da incidência de retenção de placenta permite identificar potenciais problemas futuros na reprodução e a necessidade de melhoria ou manutenção das práticas de manejo adotadas na fazenda. Como a identificação da retenção de placenta é fácil e ocorre mais precocemente que as demais desordens no pósparto, o monitoramento desse indicador pode auxiliar na melhoria do desempenho reprodutivo das fazendas.

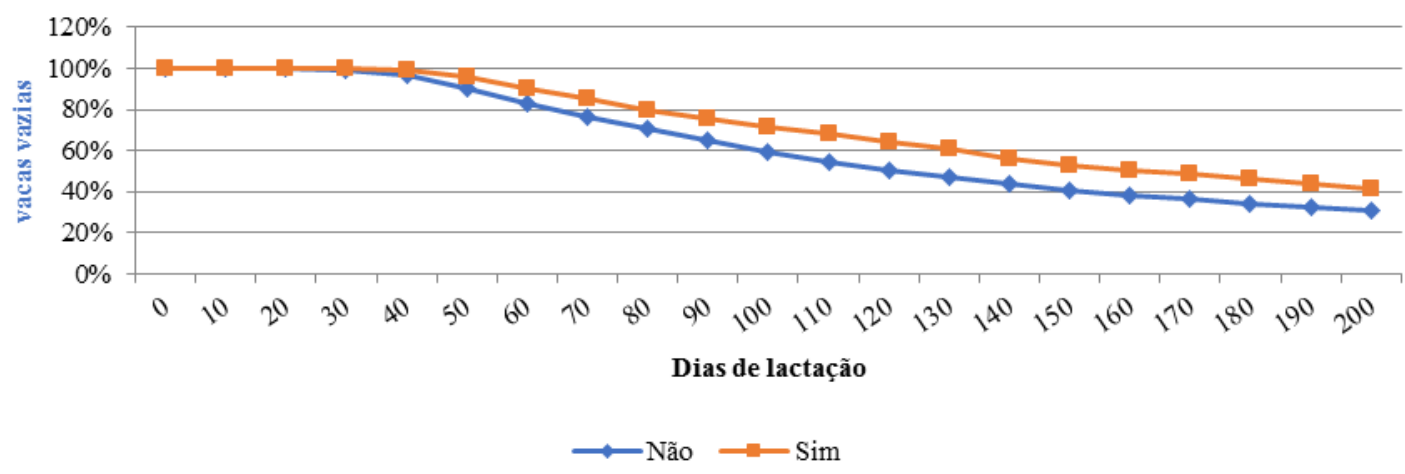

Figura 2. Curva de sobrevivência para prenhez vs. natimorto.

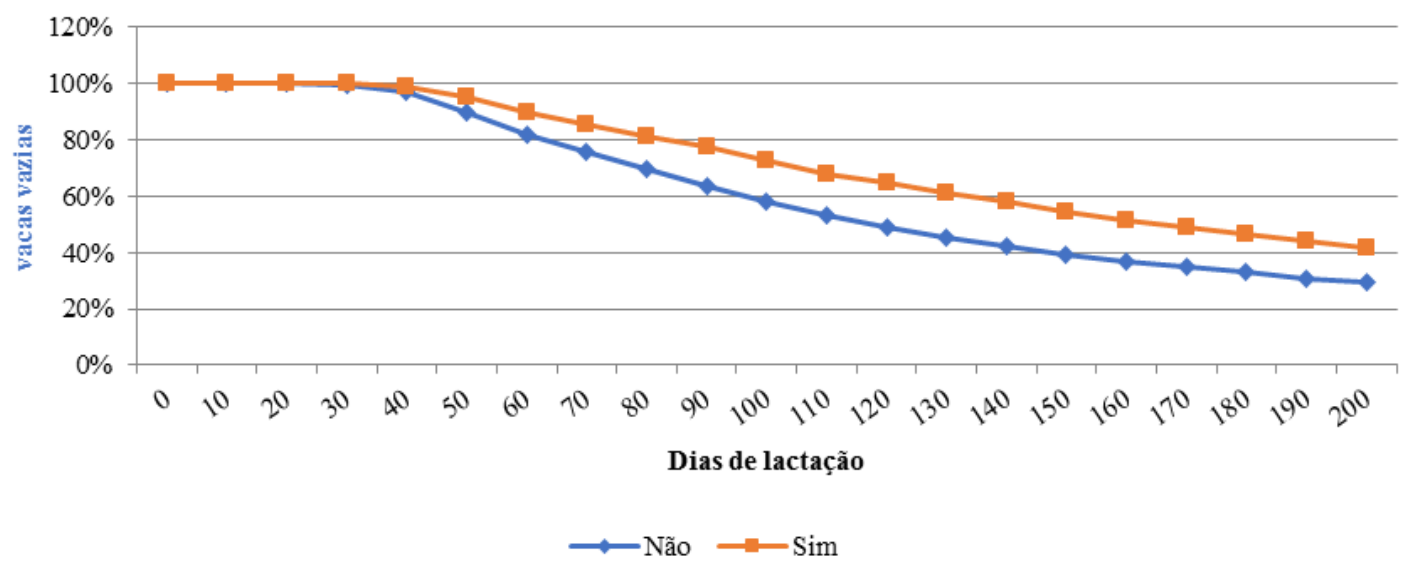

Figura 3. Curva de sobrevivência para prenhez vs. retenção de placenta.

A ordem de parto teve efeito tanto sobre a probabilidade de concepção à primeira inseminação quanto sobre a probabilidade de prenhez aos 100, 150 ou 200 DEL (Fig. 4). Vacas a partir do terceiro parto tiveram a probabilidade de concepção à primeira inseminação reduzida em $26,5 \%$ em relação às primíparas. Não houve diferença entre as primíparas e secundíparas para a probabilidade de concepção à primeira inseminação.
Alguns trabalhos observaram melhor desempenho reprodutivo de primíparas em relação às multíparas (Gröhn e Rajala-Schultz, 2000; Tenhagen et al., 2003); em outros trabalhos, observou-se menor desempenho reprodutivo de primíparas ou não se observou diferença (Rocha et al., 2001; Melendez e Pinedo, 2007). Rocha et al. (2001) observaram redução no desempenho reprodutivo de multíparas comparadas às primíparas. A queda 
no desempenho reprodutivo de multíparas pode ser explicada pela maior ocorrência de retenção de placenta e metrites (Bonneville-Hébert et al., 2011). A possível explicação para a redução no desempenho reprodutivo de vacas a partir da terceira cria é a maior incidência de doenças reprodutivas, como retenção de placenta e metrites.

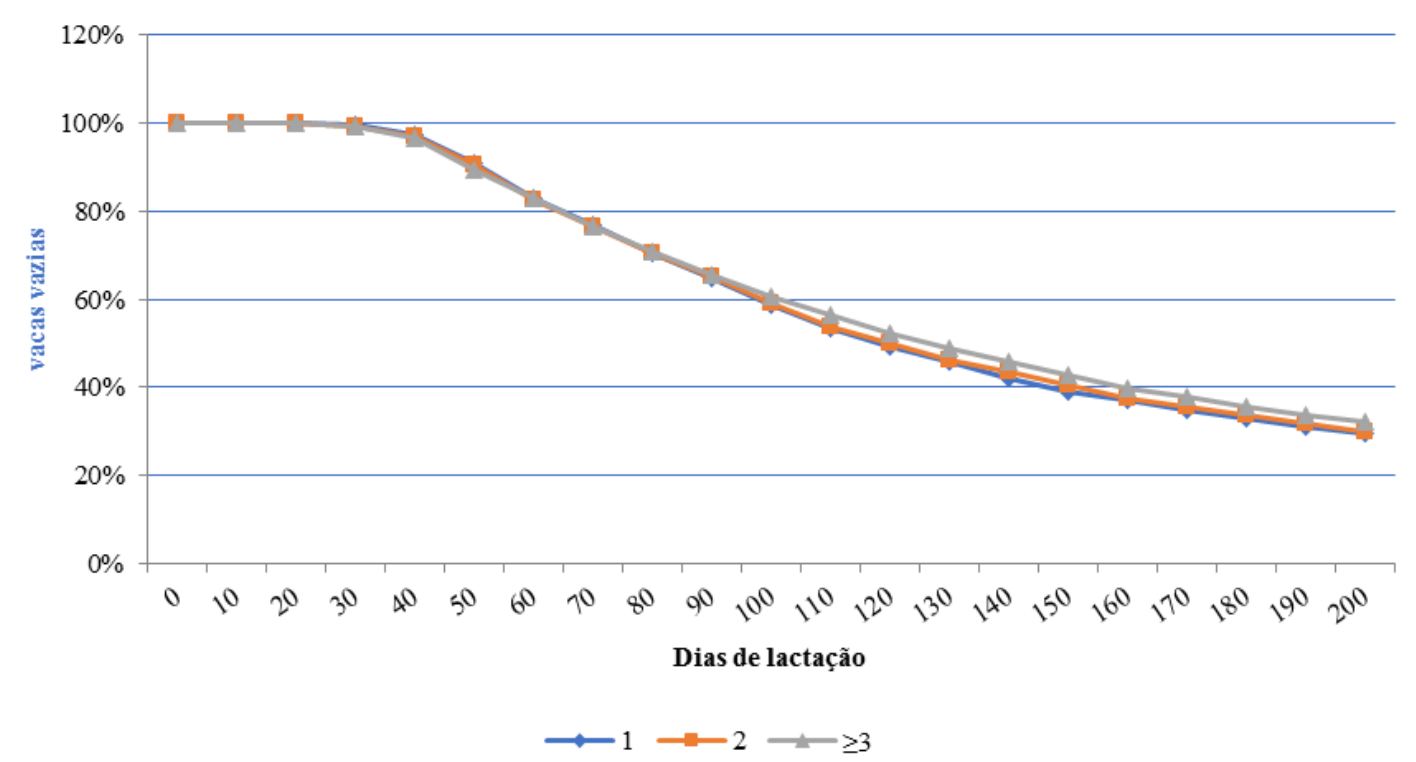

Figura 4. Curva de sobrevivência para prenhez vs. ordem de parto.

\section{CONCLUSÕES}

A taxa de concepção na primeira inseminação pós-parto e o percentual de vacas prenhes ao 100,150 e 200 DEL foram afetados pela estação do parto, estação da inseminação, ocorrência de natimorto e retenção de placenta. $\mathrm{O}$ efeito do clima no desempenho reprodutivo ficou evidente pelo efeito observado da estação do parto e estação da inseminação na concepção na primeira inseminação e percentual de vacas prenhes aos 100, 150 e 200 DEL. A saúde pósparto influencia o desempenho reprodutivo, pelos efeitos observados da retenção de placenta e natimorto. Dessa forma, pode-se pensar em ações de manejo na fazenda que permitam melhorar o desempenho reprodutivo, principalmente $\mathrm{o}$ controle do estresse térmico e a redução de doenças no pré e pós-parto. O tempo para a primeira inseminação pós-parto demonstrou ter efeito no desempenho reprodutivo, portanto quanto mais rápido for realizada a primeira inseminação, maior o percentual de vacas prenhes no decorrer da lactação.

\section{REFERÊNCIAS}

BAUMGARD, L.H.; RHOADS Jr. R.P. Effects of heat stress on postabsorptive metabolism and energetics. Аnnu. Rev. Anim. Biosci., v.1, p.311-337, 2013.

BELLO, N.M.; STEVENSON, J.S.; TEMPELMAN, R.J. et al. Invited review: milk production and reproductive performance: Modern interdisciplinary insights into an enduring axiom. J. Dairy Sci., v.95, p.5461-5475, 2012.

BERGMANN, J.A.G.; HOHENBOKEN, W.D. Prediction of fertility from calfhood traits of Angus and Simenthal heifers. J. Anim. Sci., v.70, p.26112621, 1992.

BERRY, D.P.; BUCKLEY, F.; DILLON, P. Genetic parameters for body condition score, body weight, milk yield, and fertility estimated using random regression models. J. Dairy Sci., v.86, p.3704-3717, 2003.

BERRY, D.P.; LEE, J.M.; MACDONALD, K.A. et al. Body Condition score and body weight effects on dystocia and stillbirths and consequent effects on postcalving performance. J. Dairy Sci., v.90, p.42014211, 2007. 
BONNEVILLE-HÉBERT, A.; BOUCHARD, E.; DU TREMBLAY, D. et al. Effect of reproductive disorders and parity on repeat breeder status and culling of dairy cows in Quebec. Can. J. Vet. Res., v.75, p.147-151, 2011.

DE VRIES, A.; RISCO, C.A. Trends and seasonality of reproductive performance in Florida and Georgia dairy herds from 1976 to 2002. Journal of Dairy Science, v.88, p. 3155-3165, 2005.

DRANSFIELD, M.B.G.; NEBEL, R.L.; PEARSON, L.D. et al. Timing of insemination for dairy cows identified in estrus by a radiotelemetric estrus detection system. J. Dairy Sci., v.81, p.1874-1882, 1998.

DUBOIS, P.R.; WILLIANS, D.J. Increased incidence of retained placenta associated with heat stress in dairy cows. Theriogenology, v.13, p.115-121, 1980.

GRÖHN, Y.T.; ERB, H.N.; MCCULLOCH, C.E. et al. Epidemiology of reproductive disorders in dairy cattle: associations among host characteristics, disease and production. Prev. Vet. Med., v.8, p.25-39, 1990.

GRÖHN, Y.T.; RAJALA-SCHULTZ, P.J. Epidemiology of reproductive performance in dairy cows. J. Dairy Sci., v.60-61, p.605-614, 2000.

GWAZDAUSKAS, F.C.; THATCHER, W.W.; KIDDY, C.A. et al. Hormonal pattern during heat stress following PGF2alpha-tham salt induced luteal regression in heifers. Theriogenology, v.16, p.271-285, 1981.

HANSEN, P.J. Physiological and cellular adaptations of zebu cattle to thermal stress. Animal Reproduction Science, v.82-83, p. 349-360, 2004.

HOSMER, D.W.; LEMESHOW, S. Applied logistic regression. 2.ed. New York: John Wiley and Sons, Inc., 1985. 392p.

HUANG, C.; TSURUTA, S.; BERTRAND, J.K. et al. Trends for conception rate of Holstein over time in southeastern United States. J. Dairy Sci., v.92, p.46414647, 2009.

INCHAISRI, C.; HOGEVEEN, H.; VOS, P.L.A.M. et al. Effect of milk yield characteristics, breed, and parity on success of the first insemination in Dutch dairy cows. J. Dairy Sci., v.93, p.5179-5187, 2010.

LAVEN, R.A.; PETERS, A.R. Bovine retained placenta: aetiology, pathogenesis and economic loss. Vet. Rec., v.139, p.465-471, 1996.
LeBLANC, S.J. Postpartum uterine disease and dairy herd reproductive performance: a review. Vet. J., v.176, p.102-114, 2008.

MELENDEZ, P.; PINEDO, P. The association between reproductive performance and milk yield in Chilean Holstein cattle. J. Dairy Sci., v.90, p.184-192, 2007.

NOBRE, M.M.; COELHO, S.G.; HADDAD, J.P. et al. Avaliação da incidência e fatores de risco da retenção de placenta em vacas mestiças leiteiras. Arq. Bras. Med. Vet. Zootec., v.64, p.101-107, 2012.

PAULA-LOPES, F.F.; AL-KATANANI, Y.M.; MAJEWSKI, A.C. et al. Manipulation of antioxidant status fails to improve fertility of lactating cows or survival of heat-shocked embryos. J. Dairy Sci., v.86, p.2343-2351, 2003

ROCHA, A.; ROCHA, S.; CARVALHEIRA, J. Reproductive and efficiency of inseminators in dairy farms in Portugal. Reprod. Domest. Anim., v.36, p.319-324, 2001.

SARTORI, R.; SARTOR-BERGFELT, R.; MERTENS, S.A. et al. Fertilization and Early Embryonic Development in Heifers and Lactating Cows in Summer and Lactating and Dry Cows in Winter. J. Dairy Sci., v.85, p.2803-2812, 2002.

STATISTICAL analysis system. SAS/STAT Software. Version 9.0. Cary: SAS Institute, Inc., 2006.

TEIXEIRA, A.A. Impacto da inseminação artificial em tempo fixo na eficiência reprodutiva de vacas de leite de alta produção. 2010. 69f. Dissertação (Mestrado em Reprodução Animal) - Universidade de São Paulo, São Paulo, SP.

TENHAGEN, B.A.; VOGEL, C.; DRILLICH, M. et al. Influence of stage of lactation and milk production on conception rates after timed artificial insemination following Ovsynch. Theriogenology, v.60, p.15271537, 2003.

TORRES-JÚNIOR, J.R.S.; PIRES, M.F.A.; SÁ, W.F. Effect of maternal heat-stress on follicular growth and oocyte competence in Bos indicus cattle. Theriogenology, v.69, p.155-166, 2008.

TSURUTA, S.; MISZTAL, I.; HUANG, C. et al. Bivariate analysis of conception rates and test-day milk yields in Holsteins using a threshold-linear model with random regressions. J. Dairy Sci., v.92, p.29222930, 2009. 